\title{
Implementing a Model Checking Algorithm by Adapting Existing Automated Tools
}

\author{
Bengt Jonsson Ahmed Hussain Khan \\ Joachim Parrow \\ Swedish Institute of Computer Science, Stockholm*
}

\begin{abstract}
Designs of communicating systems can be validated by checking that their behavior satisfy desirable properties expressed in a temporal logic. We describe an adaptation of a branchingtime temporal logic, CTL, to which we have given a semantics in terms of sequences of communication actions. We describe a method for checking that a communicating system satisfies a formula in the logic. The method works as follows: we first transform the communicating system to conform with the standard semantics of CTL; we then use an existing algorithm for CTL with the standard semantics, for which an implementation exists in the EMC, to check that the system satisfies a formula. The method is implemented within the framework of the Concurrency Workbench.
\end{abstract}

\section{Introduction}

An important method for ensuring the correctness of a communicating system is to check whether it satisfies a number of desirable properties. Properties, such as freedom from deadlock, mutual exclusion, delivery of messages, responsiveness, etc., can often be formulated in a temporal logic (e.g. [CES86, Pnu82, HM85, Lar88]). If the system is finite-state, it is often possible to check automatically whether it satisfies a property by so-called model checking algorithms [CES86, VW86, LP85, EL86]. Model checking has become one of the most important automatic verification methods for systems such as communication protocols, synchronization algorithms, etc.

Existing temporal logics for expressing properties of communicating systems can roughly be divided into linear-time and branching-time logics. For the branching-time logics (e.g. [CES86, RRSV87]) there are efficient (polynomial time) model checking algorithms. For the linear-time logics modelchecking algorithms require exponential time in the size of the formula [LP85, VW86].

In this paper, we shall describe a temporal logic for communicating systems, and its integration into an existing automated tool: the Concurrency Workbench (CWB) [CPS]. The CWB is an automated tool for the analysis of communicating systems, which embodies several different analysis methods. In CWB, systems are described in terms of their capability to perform (sequences of) communication actions, using Milner's Calculus of Communicating Systems [Mil80]. Our aim has been to extend CWB with an efficient model-checking facility for a temporal logic that can express properties of (sequences of) communication actions, and implement it efficiently with relatively small effort. The Concurrency Workbench contains a model checker for the $\mu$-calculus [EL86, SW89], a branching time logic. However, formulas in the $\mu$-calculus are interpreted over states, so it is difficult to express e.g. "the action $a$ happens" and related properties.

\footnotetext{
"Address: SICS, Box 1263, S-16428 Kista, SWEDEN, E-mail:bengt@sics.se, ahmed@sics.se, joachim@sics.se
} 
As our logic, we have chosen the branching-time logic CTL [CES86], for the reasons that it has an efficient model-checking algorithm, and that an efficient implementation exists in the Extended Model Checker (EMC), developed at CMU. Unfortunately, CTL expresses properties of (sequences of) states rather than communication actions, and we have therefore been forced to redefine the semantics of CTL.

More precisely, we have defined an alternative semantics for CTL in terms of sequences of communication actions (different from the standard one given in e.g. [CES86], which is given in terms of states). We have implemented a model-checking algorithm using the EMC and integrated it into the CWB. Our main implementation effort has been a transformation on communicating systems, which has the property that a given CTL formula is true of a communicating system in our semantics if and only if the CTL formula is true of the transformed system in the standard semantics. We then use the EMC to check whether the transformed system satisfies the given CTL formula.

In summary, the paper describes:

- An alternative semantics for CTL, which is given in terms of sequences of communication actions.

- A model checking method for CTL formulas in our semantics that uses a model-checking algorithm for the standard semantics. The main step in the method is a transformation between the two semantics.

- An implementation of the model-checking method obtained by combining two automated tools (CWB and EMC).

The rest of the paper is organized as follows. In the next section, we introduce labeled transition graphs as a model for communicating systems. In Section 3, we present CTL and our alternative semantics. Section 4 contains a presentation of the standard semantics of CTL. In Section 5 we describe our transformation and prove it correct. Section 6 describes our implementation, and Section 7 describes an application of it to an example: the alternating bit protocol.

\section{Labeled Transition Graphs}

In this section, we introduce labeled transition graphs.

A (finite) labeled transition graph (ltg for short) is a 4 -tuple $\left\langle S, s_{i}, \Sigma, \Delta\right\rangle$ where

$S$ is a finite set of states,

$s_{i} \in S$ is the initial state,

$\Sigma$ is a finite set of actions, not containing $\varepsilon$,

$\Delta \subseteq S \times(\Sigma \cup\{\varepsilon\}) \times S$ is a labeled transition relation.

We use $s, s^{\prime}$, etc. to range over states and $\alpha$ to range over $\Sigma \cup\{\varepsilon\}$. A labeled transition in $\Delta$ is a triple, written $s \stackrel{\alpha}{\longrightarrow} s^{\prime}$. A state $s \in S$ is called terminal if there is no transition $s \stackrel{\alpha}{\longrightarrow} s^{\prime}$ from $s$ in $\Delta$ for any $s^{\prime}$.

Intuitively, a state represents the possible future behavior of the ltg. The labeled transition relation describes how the state can change while performing actions. The labeled transition $s \stackrel{\alpha}{\longrightarrow} s^{\prime}$ means that when the $\operatorname{ltg}$ is in state $s$, then its state can change to $s^{\prime}$ while the action $\alpha$ is performed. The symbol $\varepsilon$ denotes a "dummy action" which is not in $\Sigma$. It is meant to signify that "no action occurs". 
Ltg:s can be represented graphically. Figure 1 represents an ltg which first performs either the action $a$ or $c$ and thereafter $b$ or $d$. This $\operatorname{ltg}$ is the tuple $\left\langle S, s_{i}, \Sigma, \Delta\right\rangle$ where

$S=\left\{s_{i}, s_{1}, s_{2}, s_{3}\right\}$

$\Sigma=\{a, b, c, d\}$,

$\Delta=\left\{s_{i} \stackrel{a}{\longrightarrow} s_{1}, s_{i} \stackrel{c}{\longrightarrow} s_{2}, s_{1} \stackrel{b}{\longrightarrow} s_{3}, s_{2} \stackrel{d}{\longrightarrow} s_{3}\right\}$

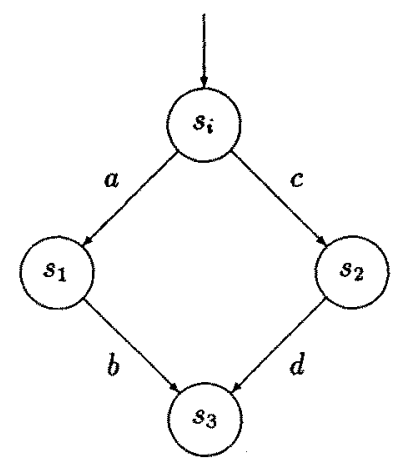

Figure 1: A labeled transition graph

\section{An Alternative Semantics for CTL}

In this section, we present the syntax of the temporal logic CTL of Clarke, Emerson and Sistla [CES86]. The semantics of CTL is defined in terms of Kripke structures. In Section 4 we give an account of this semantics of CTL. In this section, we present our alternative semantics for CTL, which is given in terms actions rather than states.

Formulas in CTL are built from atomic propositions, propositional logic connectives and temporal operators. When interpreting CTL on ltg's, it is natural to let the set of atomic propositions be the set of actions of the $1 \mathrm{tg}$. We therefore assume a set $\Sigma$ of actions, not containing $\varepsilon$, and let the set of atomic propositions be $\Sigma$. The set of CTL formulas is defined inductively as follows:

- Each atomic proposition is a CTL formula

- If $f_{1}$ and $f_{2}$ are CTL formulas, then so are $\neg f_{1}, f_{1} \wedge f_{2}, A X\left(f_{1}\right), E X\left(f_{1}\right), A\left[f_{1} U f_{2}\right]$, $E\left[\begin{array}{lll}f_{1} & \cup & f_{2}\end{array}\right]$

We shall use $f, f_{1}$, etc. to range over CTL formulas. The propositional connectives $\neg$ and $\wedge$ have their usual meanings. As usual, the connectives $\vee$ and $\longrightarrow$ can be defined in terms of these. The temporal operators $A X$ and $E X$ are next-time operators; intuitively, the formula $A X\left(f_{1}\right)$ means that $f_{1}$ holds for every transition following the current one whereas the formula $E X\left(f_{1}\right)$ means that $f_{1}$ holds for some transition following the current one. The operator $U$ is the until operator. Intuitively $A\left[\begin{array}{lll}f_{1} & U & f_{2}\end{array}\right]$ means that every sequence of transitions following the current one has an initial prefix whose last transition satisfies $f_{2}$ and whose other transitions satisfy $f_{1}$. Intuitively, $E\left[f_{1} U f_{2}\right]$ means that there is a sequence of transitions following the current one that has an initial prefix whose last transition satisfies $f_{2}$ and whose other transitions satisfy $f_{1}$. 
We define the truth of CTL-formulas for transition graphs by a satisfaction relation

$$
\langle\alpha, s\rangle \models_{T G} f
$$

which intuitively means that the formula $f$ is true when the $\operatorname{ltg} T G$ performs a transition with the action $\alpha$, leading to the state $s$.

Let $T G$ be a labeled transition graph without terminal states, and let $s_{0}$ be a state of $T G$. A path from $s_{0}$ in $T G$ is an infinite sequence

$$
s_{0} \stackrel{\alpha_{1}}{\longrightarrow} s_{1} \stackrel{\alpha_{2}}{\longrightarrow} \ldots \stackrel{\alpha_{n}}{\longrightarrow} s_{n} \stackrel{\alpha_{n+1}}{\longrightarrow} \cdots
$$

of transitions of $T G$.

Let $T G$ be the $\operatorname{ltg}\left\langle S, s_{i}, \Sigma, \Delta\right\rangle$, let $s_{0} \in S, \alpha_{0} \in \Sigma \cup\{\varepsilon\}$, and $\alpha \in \Sigma$. The satisfaction relation is defined recursively as follows.

$$
\begin{array}{ll}
\left\langle\alpha_{0}, s_{0}\right\rangle \models_{T G} \alpha & \text { iff } \alpha_{0}=\alpha \\
\left\langle\alpha_{0}, s_{0}\right\rangle \models_{T G} \neg f & \text { iff not }\left\langle\alpha_{0}, s_{0}\right\rangle \equiv_{T G} f \\
\left\langle\alpha_{0}, s_{0}\right\rangle \models_{T G} f_{1} \wedge f_{2} & \text { iff }\left\langle\alpha_{0}, s_{0}\right\rangle \models_{T G} f_{1} \text { and }\left\langle\alpha_{0}, s_{0}\right\rangle \models_{T G} f_{2} \\
\left\langle\alpha_{0}, s_{0}\right\rangle \models_{T G} A X(f) & \text { iff for all transitions } s_{0} \stackrel{\alpha_{1}}{\longrightarrow} s_{1} \in \Delta \text { it holds that }\left\langle\alpha_{1}, s_{1}\right\rangle \models_{T G} f \\
\left\langle\alpha_{0}, s_{0}\right\rangle \models_{T G} E X(f) & \text { iff for some transition } s_{0} \stackrel{\alpha_{1}}{\longrightarrow} s_{1} \in \Delta \text { it holds that }\left\langle\alpha_{1}, s_{1}\right\rangle \equiv_{T G} f \\
\left\langle\alpha_{0}, s_{0}\right\rangle \models_{T G} A\left[f_{1} U f_{2}\right] & \text { iff for all paths } s_{0} \stackrel{\alpha_{1}}{\longrightarrow} s_{1} \stackrel{\alpha_{2}}{\longrightarrow} \cdots \text { from } s_{0} \text { in } T G \text { there exists } \\
& \text { an } i \geq 0 \text { such that } \\
& (1)\left\langle\alpha_{i}, s_{i}\right\rangle \models_{T G} f_{2}, \text { and } \\
& (2) \text { for all } j \text { with } 0 \leq j<i \text { it holds }\left\langle\alpha_{j}, s_{j}\right\rangle \models_{T G} f_{1} .
\end{array}
$$

$\left\langle\alpha_{0}, s_{0}\right\rangle \models_{T G} E\left[\begin{array}{lll}f_{1} & U & f_{2}\end{array}\right] \quad$ iff for some path $s_{0} \stackrel{\alpha_{1}}{\longrightarrow} s_{1} \stackrel{\alpha_{2}}{\longrightarrow} \cdots$ from $s_{0}$ in $T G$, there exists an $i$ such that

(1) $\left\langle\alpha_{i}, s_{i}\right\rangle \equiv_{T G} f_{2}$, and

(2) for all $j$ with $0 \leq j<i$ it holds $\left\langle\alpha_{j}, s_{j}\right\rangle \equiv_{T G} f_{1}$.

If $T G$ is an $\operatorname{ltg}$ with initial state $s_{i}$, then we define

$$
\equiv_{T G} f \quad \text { iff } \quad\left\langle\varepsilon, s_{i}\right\rangle \equiv_{T G} f
$$

This implies for instance that if $\alpha$ is any action in $\Sigma$, then $\models_{T G} \alpha$ is false for any transition graph $T G$.

The truth of CTL formulas for arbitrary ltg:s is defined as follows. For an arbitrary transition graph $T G=\left\langle S, s_{i}, \Sigma, \Delta\right\rangle$ define $\widehat{T G}$ to be $\left\langle S \cup\left\{s_{f}\right\}, s_{i}, \Sigma, \Delta \cup \Delta^{\prime}\right\rangle$, where $s_{f}$ is an extra "final" state not in $S$, and $\Delta^{\prime}$ contains the transitions $s \stackrel{\varepsilon}{\longrightarrow} s_{f}$ for each terminal $s$ of $T G$ and the transition $s_{f} \stackrel{\varepsilon}{\longrightarrow} s_{f}$. We now define

$$
\models_{T G} f \text { iff } \equiv_{\widehat{T G}} f
$$

We shall also use the following defined CTL operators:

$$
\begin{aligned}
& A F(f) \equiv A[\text { true } U f] \\
& E F(f) \equiv E[\text { true } U f] \\
& A G(f) \equiv \neg E F(\neg f) \\
& E G(f) \equiv \neg A F(\neg f)
\end{aligned}
$$

Intuitively, $A F(f)(E F(f))$ means that $f$ holds for some action along every (some) future path. Intuitively, $A G(f)(E G(f))$ means that $f$ holds for all actions along every (some) future path.

Example 1 Let $T G$ be the labeled transition graph in Figure 1. We have

$$
\begin{aligned}
\left\langle a, s_{1}\right\rangle & \equiv_{T G} a \\
& \equiv_{T G} A X(a \vee c) \\
& \equiv_{T G} \quad A F(b \vee c)
\end{aligned}
$$




\section{$4 \quad$ Kripke Structures}

In order to describe our implementation of a model checking algorithm for CTL, as defined in Section 3, we must introduce Kripke structures and present the standard interpretation of CTL formulas in such structures, as formulated in e.g. [CES86].

Assume a set $A P$ of atomic propositions, ranged over by $p$. A (finite) Kripke Structure is a quadruple $\left\langle T, t_{i}, R, P\right\rangle$ where

$T$ is a finite set of states

$t_{i} \in T$ is an initial state

$R \subseteq T \times T$, is a set of (unlabeled) transitions. The relation $R$ must be total, i.e., $(\forall t \in T)\left(\exists t^{\prime} \in T\right)\left[\left\langle t, t^{\prime}\right\rangle \in R\right]$.

$P$ is an assignment of atomic propositions to states, i.e., $P: T \rightarrow 2^{A P}$.

We use $t, t^{\prime}$, etc. to range over states in Kripke structures. A transition is a pair, which we denote $\left\langle t, t^{\prime}\right\rangle$. A path from a state $t_{0}$ in a Kripke structure $K S$ is an infinite sequence $\left(t_{0}, t_{1}, t_{2}, \ldots\right)$ of states where each consecutive pair $\left\langle t_{i-1}, t_{i}\right\rangle$ is a transition of $K S$.

We use the same syntax for CTL formulas as in Section 3, with $A P$ for the set of atomic propositions. The standard semantics of CTL formulas is given by a satisfaction relation

$$
t_{0} \vDash_{K S} f
$$

which intuitively states that the CTL formula $f$ is true for the state $t_{0}$ in the Kripke structure $K S$. The relation is defined inductively as follows:

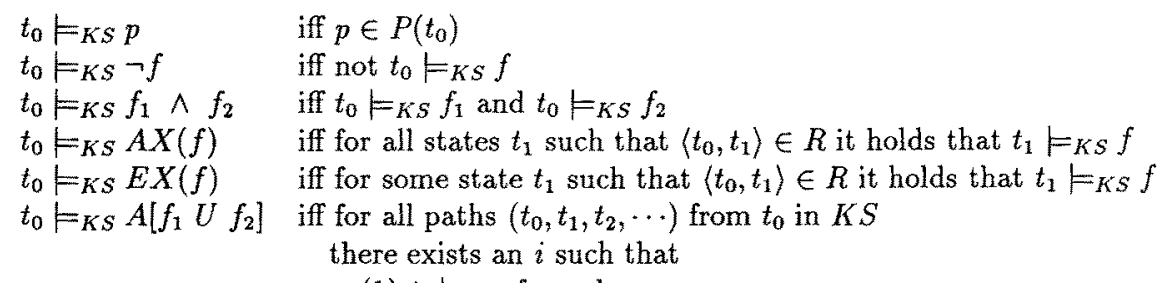

(1) $t_{i} \models_{K S} f_{2}$, and

(2) for all $j$ with $0 \leq j<i$ it holds $t_{j} \vDash_{K S} f_{1}$

$t_{0} F_{K S} E\left[f_{1} U f_{2}\right]$ iff for some path $\left(t_{0}, t_{1}, t_{2}, \cdots\right)$ from $t_{0}$ in $K S$

there exists an $i$ such that

(1) $t_{i} F_{K s} f_{2}$, and

(2) for all $j$ with $0 \leq j<i$ it holds $t_{j} \models_{K S} f_{1}$

We define $\models_{K S} f$ to mean that $t_{i} \models_{K S} f$, where $t_{i}$ is the initial state of $K S$. The operators, $A F$, $E F, A G, E G$ are defined as in Section 3 .

\section{Transformation from Transition Graphs to Kripke Struc- tures}

In this section, we describe our transformation of a labeled transition graph (ltg) into a Kripke structure, and prove that this transformation is compatible with the definition of truth of CTLformulas in the two semantics that have been given. We observe that the semantics in Section 3 is given in terms of transitions and actions, whereas the semantics in Section 4 is given in terms of 
states. The idea of the transformation is therefore to transform transitions of the ltg into states of a Kripke structure, and to let the transitions of the Kripke structure reflect how transitions of the $1 \mathrm{tg}$ can follow each other.

Let $T G=\left\langle S, s_{i}, \Sigma, \Delta\right\rangle$ be a labeled transition graph. Let $A P$, the set of atomic propositions, be $\Sigma$. The Kripke structure corresponding to $T G$, written $k s(T G)$, is the tuple $\left\langle T, t_{i}, R, P\right\rangle$, where

$T=\Delta \cup\left\{t_{i}, t_{f}\right\}$, where $t_{i}$ and $t_{f}$ are distinct states, not in $\Delta$.

$$
\begin{aligned}
R= & \left\{\left\langle s \stackrel{\alpha}{\longrightarrow} s^{\prime}, s^{\prime} \stackrel{\alpha^{\prime}}{\longrightarrow} s^{\prime \prime}\right\rangle \mid s \stackrel{\alpha}{\longrightarrow} s^{\prime}, s^{\prime} \stackrel{\alpha^{\prime}}{\longrightarrow} s^{\prime \prime} \in \Delta\right\} \\
& \cup\left\{\left\langle t_{i}, s_{i} \stackrel{\alpha}{\longrightarrow} s^{\prime}\right\rangle \mid s_{i} \stackrel{\alpha}{\longrightarrow} s^{\prime} \in \Delta\right\} \\
& \cup\left\{\left\langle s \stackrel{\alpha}{\longrightarrow} s^{\prime}, t_{f}\right\rangle \mid s \stackrel{\alpha}{\longrightarrow} s^{\prime} \in \Delta \text { such that } s^{\prime} \text { is terminal in } T G\right\} \\
& \cup\left\{\left\langle t_{f}, t_{f}\right\rangle\right\}
\end{aligned}
$$

$P$ is defined by

$$
\begin{aligned}
& P\left(s \stackrel{\alpha}{\longrightarrow} s^{\prime}\right)=\{\alpha\} \text { for } s \stackrel{\alpha}{\longrightarrow} s^{\prime} \in \Delta, \text { and } \\
& P\left(t_{i}\right)=P\left(t_{f}\right)=\emptyset .
\end{aligned}
$$

Intuitively, the states in $T$ are the transitions of $T G$ plus an extra initial state $t_{i}$ and final state $t_{f}$. Due to the requirement that $R$ be total, we must in $R$ add a self-loop at $t_{f}$.

Example 2 We can represent a Kripke structure graphically. In Figure 2 is shown a Kripke structure which results from transforming the ltg shown in Figure 1. This Kripke structure has one state for each transition of the $1 \mathrm{tg}$, and in addition an initial and a final state. Within each state, we show the set of atomic propositions that are assigned to it.

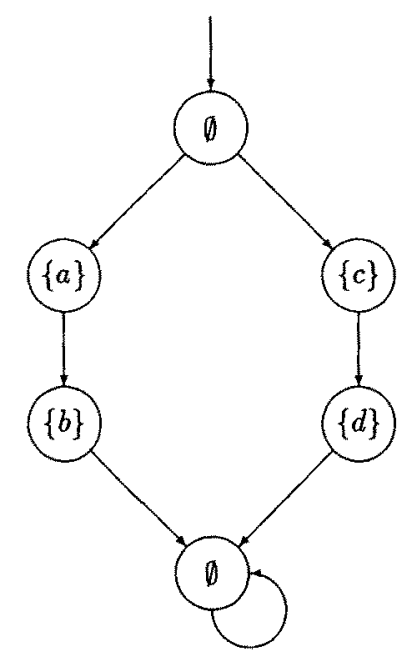

Figure 2: A Kripke structure resulting from transformation

The main theoretical result of this paper is that this transformation is compatible with the definition of truth of CTL formulas.

Theorem 1 For all transition graphs $T G$ and CTL formulas $f$ it holds that

$$
\models_{T G} f \quad \text { iff } \quad \models_{k s(T G)} f
$$


Proof: For a transition graph $T G$, define $\overparen{T G}$ to be $\widehat{T G}$ (as defined in Section 3) with an extra state $s_{e}$ and an extra transition $s_{e} \stackrel{s}{\longrightarrow} s_{i}$. Define a relation $\sim$ between transitions of $\overline{T G}$ and states of $k s(T G)$ as follows: For a state $t$ of $k s(T G)$ not being $t_{i}$ or $t_{f}$, let $s \stackrel{\alpha}{\longrightarrow} s^{\prime} \sim t$ iff $t=s \stackrel{\alpha}{\longrightarrow} s^{\prime}$, and let $s_{e} \stackrel{\varepsilon}{\longrightarrow} s_{i} \sim t_{i}$, and $s \stackrel{\varepsilon}{\longrightarrow} s_{f} \sim t_{f}$ whenever $s$ is a terminal state of $T G$ or $s=s_{f}$. From the definition of $k s(T G)$, it follows that for each finite or infinite sequence

$$
s_{0} \stackrel{\alpha_{1}}{\longrightarrow} s_{1} \stackrel{\alpha_{2}}{\longrightarrow} \ldots \stackrel{\alpha_{n}}{\longrightarrow} s_{n} \stackrel{\alpha_{n+1}}{\longrightarrow} \ldots
$$

of transitions of $\widetilde{T G}$ there is a path $\left(t_{1}, t_{2}, \ldots, t_{n}, \ldots\right)$ of $k s(T G)$, such that $s_{m-1} \stackrel{\alpha}{\longrightarrow} s_{m} \sim t_{m}$ for all $s_{m-1} \stackrel{\alpha}{\longrightarrow} s_{m}$ in the first sequence. Conversely, for each finite or infinite sequence $\left(t_{1}, t_{2}, \ldots, t_{n}, \ldots\right)$ of states of $k s(T G)$, where each consecutive pair $\left\langle t_{i-1}, t_{i}\right\rangle$ is a transition of $k s(T G)$, there is a path

$$
s_{0} \stackrel{\alpha_{1}}{\longrightarrow} s_{1} \stackrel{\alpha_{2}}{\longrightarrow} \ldots \stackrel{\alpha_{n}}{\longrightarrow} s_{n} \stackrel{\alpha_{n+1}}{\longrightarrow} \ldots
$$

of $\widetilde{T G}$ such that $s_{m-1} \stackrel{\alpha}{\longrightarrow} s_{m} \sim t_{m}$ for all $t_{m}$ in the first sequence.

Next observe that if $s \stackrel{\alpha}{\longrightarrow} s^{\prime} \sim t$ and $\alpha^{\prime} \in \Sigma$ then $\left\langle\alpha, s^{\prime}\right\rangle \equiv_{T G^{\prime}} \alpha^{\prime}$ iff $t \models_{k s\left(T G^{\prime}\right)} \alpha^{\prime}$. Using this observation and the above correspondence between sequences of transitions of $\overline{T G}$ and sequences of states of $k s(T G)$, we can by induction over $f$ prove that for all transitions $s \stackrel{\alpha}{\longrightarrow} s^{\prime}$ of $\widetilde{T G}$ and states $t$ of $k s(T G)$ such that $s \stackrel{\alpha}{\longrightarrow} s^{\prime} \sim t$ we have $\left\langle\alpha, s^{\prime}\right\rangle \models_{\widehat{T G}} f$ iff $t \vDash_{k s(T G)} f$. Theorem 1 can now be proven as follows:

$$
\models_{T G} f \text { iff }\left\langle\varepsilon, s_{i}\right\rangle \models_{\widehat{T G}} f \text { iff } \quad t_{i} \models_{k s(T G)} f \text { iff } \quad \models_{k s(T G)} f
$$

\section{Implementation}

In this section, we describe the architecture of our implementation of an algorithm for checking that a labeled transition graph satisfies a CTL formula. Our implementation is a combination of two existing automatic tools: the Concurrency Workbench (CWB), and the Extended Model-Checker (EMC). We use CWB for generating transition graphs from CCS expressions. A graphical interface is under development. We will not further deal with that aspect in this paper. We use the existing model checking algorithm of EMC to check transition graphs after they have been transformed.

We have implemented a converter in Standard ML, which performs the transformation from transition graphs to Kripke structures described in section 5 . The architecture of the resulting system is shown in Figure 3.

\section{Application: the Alternating Bit Protocol}

In this section, we show by an example how our system can be used. We have chosen the Alternating Bit Protocol [BSW69], for the reason that this example is widely used as a test case in the literature, and that it is described in [CES86] where it is described how CTL formulas with the standard interpretation are checked using EMC.

The Alternating Bit Protocol consists of two processes, a Sender and a Receiver, which alternately exchange messages. The Sender accepts messages from the environment; the purpose of the protocol is to transfer these messages to the receiver and then deliver them to the environment.

Messages from the Sender to the Receiver are data messages and messages from the Receiver to the Sender are acknowledgments. Messages may become garbled during transmission, but we assume that each message is encoded so that garbled messages can be detected. 


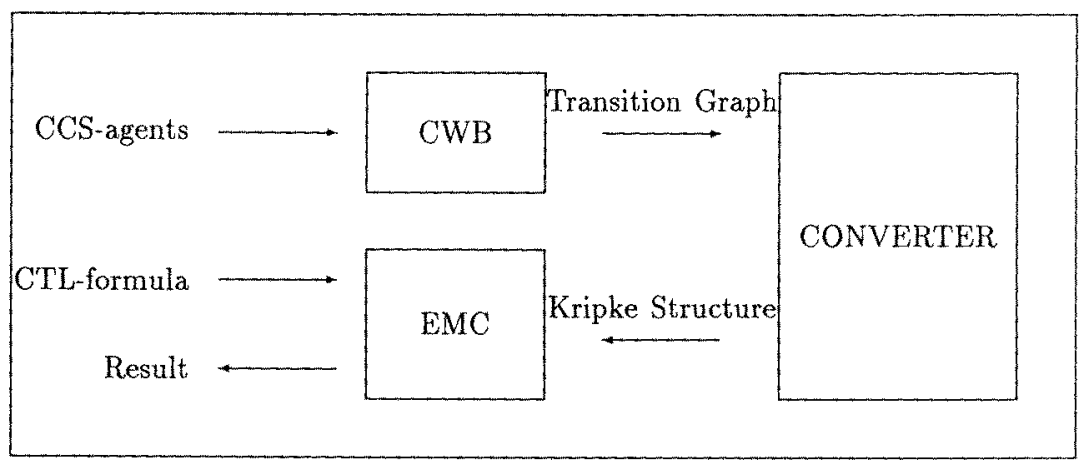

Figure 3: Architecture of our implementation

The Sender sends data messages containing data and a sequence number. The sequence number is either 0 or 1 . The Receiver waits for a data message with a particular sequence number. If a message with the correct sequence number arrives, the Receiver returns an acknowledgment with the same sequence number; thereafter both Sender and Receiver change sequence numbers. If a data message with the wrong sequence number or a garbled message arrives, then an acknowledgment with the sequence number that was not expected.

In [CES86], the protocol is described in CSP. We have adapted this version of the protocol as a labeled transition graph in Figure 4. We assume that messages from the environment are either 0 or 1. The action si with $i$ either 0 or 1 represents the receipt of message $i$ from the environment, dij is a data message with message $i$ and sequence number $j$, The message $a i$ is an acknowledgment with sequence number $i$, and $r i$ represents the delivery of message $i$ to the environment. The actions de and ae represent garbled messages.

Following [CES86], the CSP mode of communication is employed, in which a message is sent and received in one atomic action. Note that for each message exchange between the Sender and Receiver there is a corresponding garbled message exchange, modeling corruption of the message.

The labeled transition graphs in Figure 4 can now be entered in to the Concurrency Workbench, which from the two processes generates one global Itg representing the behavior of the entire protocol. This generation couples transitions of the two processes. For this global $\mathrm{ltg}$ we can now test CTL formulas such as:

$$
\begin{aligned}
& A G[r \longrightarrow A X[A(\neg r U s)]] \\
& A G[s 0 \longrightarrow A X[A((\neg s \wedge \neg r 1) U r 0)]]
\end{aligned}
$$

where we have used $s$ as a shorthand for $(s 0 \vee s 1)$ and $r$ as a shorthand for $(r 0 \vee \vee 1)$. These formulas are adapted from [CES86]. Here the first formula intuitively states that after a receive action eventually a send action will be performed, and in between these actions no other receive action will be performed. The second formula intuitively states that after a send action with message 0 , eventually a receive action with message 0 will be performed, and in between these actions no other action that involves the environment will be performed.

Due to the fact that messages can be garbled continuously, these formulas are not satisfied by the protocol. One needs an extra assumption about the protocol which ensures that messages are not continuously garbled from some point on. Such assumptions can be entered into the EMC in the form of fairness requirements. In EMC, fairness assumptions must be of the form "formula $f$ holds infinitely often on every path", where $f$ is a CTL formula without any temporal operators ( $A X$, 


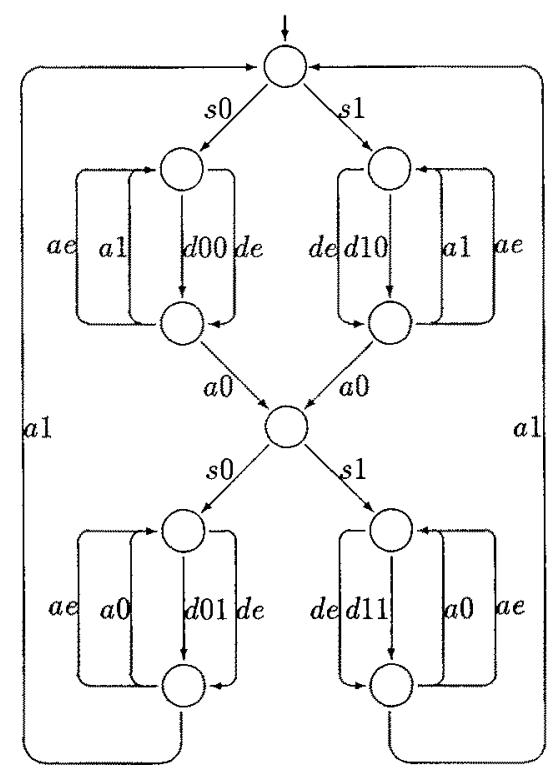

Sender

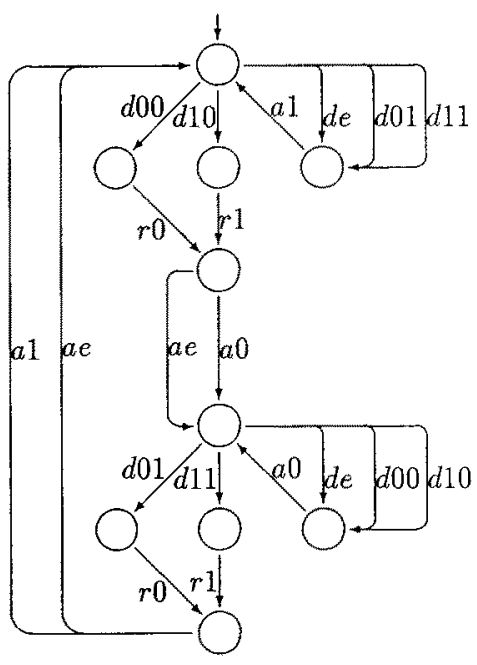

Receiver

Figure 4: the Alternating Bit Protocol

$E X, A U$, or $E U$ ). In this example we can, following the treatment in [CES86], introduce the fairness assumption "predicate $(s 0 \vee s 1)$ holds infinitely often on every path", which implies that the behavior of the protocol is cyclic. Under this fairness assumption the above formulas hold.

\section{Conclusion}

We have presented a temporal logic for communicating systems, and its integration into an existing automated tool: the Concurrency Workbench (CWB) [CPS]. We have illustrated how one can change the semantics of an existing logic, and how one can adapt existing automated tools to implement decision procedures such as model checking.

Interesting future work would be to use our system for comparing different implementations of model checking algorithms for branching time logics with respect to convenience and performance. The implementations that we have in mind are the EMC, XESAR [RRSV87], and the implementation of the mu-calculus that exists in the CWB.

\section{Acknowledgments}

We are grateful to Rance Cleaveland, Fredrik Orava, Colin Stirling, and David Walker for comments and fruitful discussions, and to Ed Clarke for helping us understand the EMC. 


\section{References}

[BSW69] K. Bartlett, R. Scantlebury, and P. Wilkinson. A note on reliable full-duplex transmissions over half duplex lines. Communications of the ACM, 2(5):260-261, 1969.

[CES86] E. M. Clarke, E. A. Emerson, and A. P. Sistla. Automatic verification of finite-state concurrent systems using temporal logics specifications. ACM TOPLAS, 8(2):244-263, April 1986.

[CPS] R. Cleaveland, J. G. Parrow, and B. Steffen. The concurrency workbench: Operating instructions. University of Edinburgh, Laboratory for Foundations of Computer Science, Technical Note 10, September 1988.

[EL86] E.A. Emerson and C.-L. Lei. Efficient model checking in fragments of the propositional mu-calculus. In Proc. Symp. on Logics in Computer Science, pages 267-278, 1986.

[HM85] Matthew Hennessy and Robin Milner. Algebraic laws for nondeterminism and concurrency. J. ACM, 32(1):137-161, 1985.

[Lar88] K. G. Larsen. Proof systems for Hennessy-Milner logic with recursion. In Proc. CAAP 1988, volume 299 of Lecture Notes of Computer Science. Springer Verlag, 1988.

[LP85] O. Lichtenstein and A. Pnueli. Checking that finite state concurrent programs satisfy their linear specification. In Proc. 12th ACM Symp. on Principles of Programming Languages, pages $97-107,1985$.

[Mil80] R. Milner. A Calculus of Communicating Systems, volume 92 of Lecture Notes of Computer Science. Springer Verlag, 1980.

[Pnu82] A. Pnueli. The temporal semantics of concurrent programs. Theoretical Computer Science, $13: 45-60,1982$.

[RRSV87] J. Richier, C. Rodriguez, J. Sifakis, and J. Voiron. Verification in XESAR of the sliding window protocol. In Proceedings of the 7th IFIP Workshop on Protocol Specification, Testing and Verification. North-Holland, 1987.

[SW89] C. Stirling and D. J. Walker. Local model checking in the modal $\mu$-calculus. In Diaz and Orejas, editors, TAPSOFT' 89 , volume 351 of Lecture Notes of Computer Science, pages 369-383. Springer Verlag, 1989.

[VW86] M. Y. Vardi and P. Wolper. An automata-theoretic approach to automatic program verification. In Symp. on Logic in Computer Science, pages 332-344, June 1986. 